\title{
Pratiques transnationales des Capverdiens au Portugal et des Portugais en Suisse
}

José Carlos Marques et Pedro Góis

\section{(2) OpenEdition}

Édition électronique

URL : https://journals.openedition.org/remi/4592

DOI : $10.4000 /$ remi.4592

ISSN : $1777-5418$

Éditeur

Université de Poitiers

\section{Édition imprimée}

Date de publication : 1 novembre 2008

Pagination : 147-165

ISBN : 978-2-911627-49-0

ISSN : 0765-0752

Référence électronique

José Carlos Marques et Pedro Góis, « Pratiques transnationales des Capverdiens au Portugal et des Portugais en Suisse », Revue européenne des migrations internationales [En ligne], vol. 24 - n² | 2008, mis en ligne le 01 novembre 2011, consulté le 14 avril 2022. URL : http://journals.openedition.org/ remi/4592 ; DOI : https://doi.org/10.4000/remi.4592 


\title{
Pratiques transnationales des Capverdiens au Portugal et des Portugais en Suisse
}

\author{
José Carlos MARQUES* et Pedro GÓIS**
}

\section{LE PORTUGAL, UN PAYS D'ÉMIGRATION ET D'IMMIGRATION}

Il est bien connu que le Portugal a une longue tradition d'émigration et une courte histoire d'immigration. Mais ce qui est moins connu, c'est que, depuis quelques décennies, ces deux flux migratoires coexistent. Ils sont imbriqués d'une manière originale et l'évolution de l'un et de l'autre se déroule de façon concomitante. La figure suivante résume les phases des migrations au Portugal au cours des dernières décennies.

Le système migratoire européen et le système migratoire lusophone fonctionnent comme des vases communicants (Góis et Marques, 2006). Il s'agit en outre de mouvements migratoires qui présentent quelques similitudes au niveau de l'insertion sur le marché du travail dans les pays de destination, du mode de migration et de la durée du séjour dans le pays d'accueil. Ces facteurs ont été identifiés comme étant associés au développement de certaines formes de liaison des migrants avec leurs pays d'origine. Finalement, il est à souligner dans le contexte du présent texte, qu'ils présentent des caractéristiques semblables en ce qui concerne les domaines, les types et l'intensité des pratiques qu'ils développent, notamment, par rapport au pays d'origine et/ou avec des pays tiers.

* Centro de Estudos Sociais da Universidade de Coimbra e Universidade Católica Portuguesa (Viseu) : jclaranjo@sapo.pt

** Centro de Estudos Sociais da Universidade de Coimbra e Faculdade de Belas Artes da Universidade do Porto : pedrogois@netcabo.pt 
Figure 1 : Différentes phases et types des migrations au Portugal

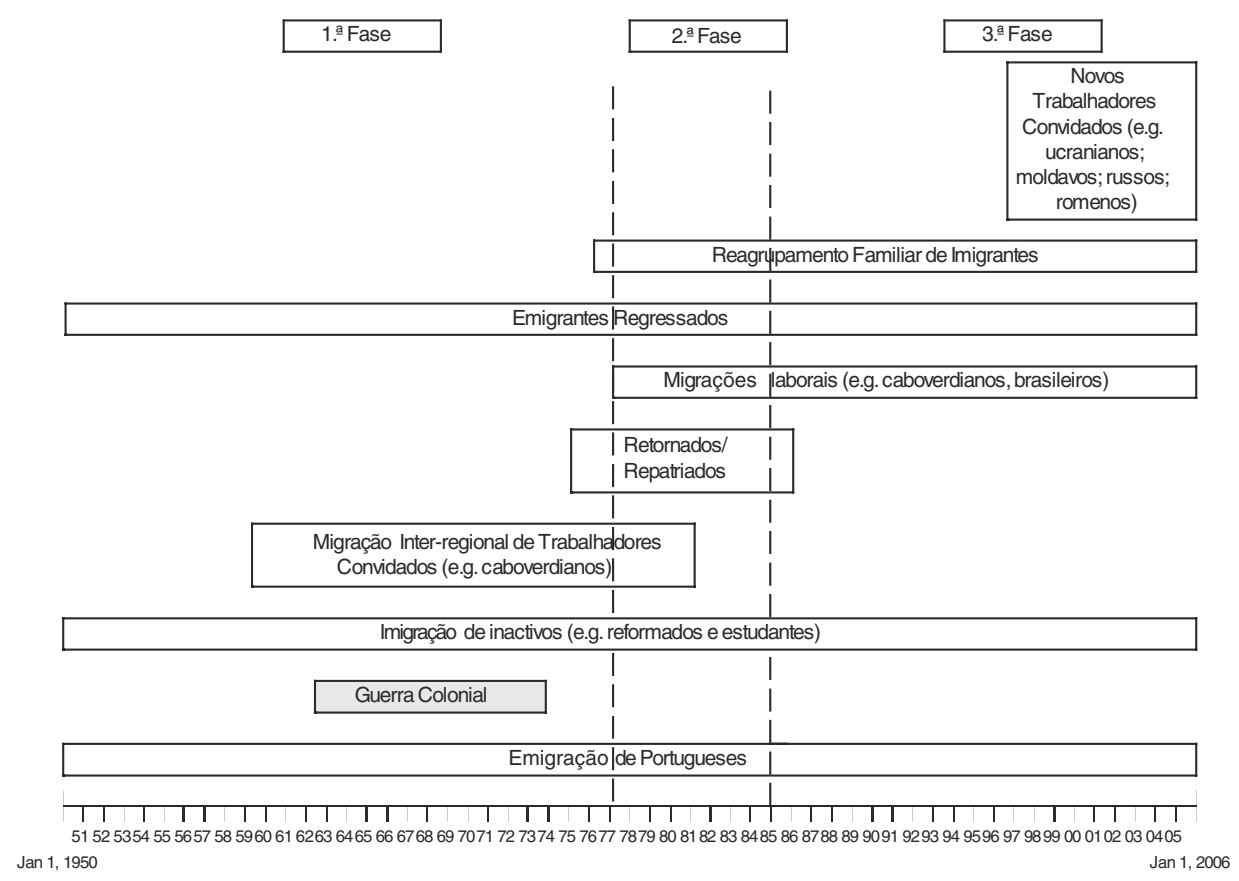

Cet article a pour objectif de présenter les différentes formes de rapport des migrants à leur pays d'origine, en identifiant celles qui peuvent être considérées comme des activités transnationales ${ }^{1}$. L'analyse des deux mouvements migratoires, de leurs ressemblances et différences dans diverses sphères de la vie est un préalable nécessaire à l'identification des pratiques transnationales développées par les groupes de migrants étudiés. Le contexte dans lequel les flux migratoires ont lieu et l'ampleur du mouvement influencent l'émergence et l'évolution de ces pratiques : ainsi, cet article commence par présenter brièvement l'histoire et la dynamique des deux migrations. Après une discussion de la notion de transnationalisme, nous portons notre attention sur la description des activités réalisées par les migrants et observées entre 2005 et 2007, en établissant une liaison entre les deux flux migratoires et en les faisant dialoguer entre eux. Enfin nous dressons un bilan des formes de transnationalisme relevées sur le terrain et nous nous interrogeons sur les limites d'une approche qualitative synchronique pour l'étude des pratiques transnationales et nous plaidons pour une perspective comparée, s'appuyant sur des outils qualitatifs et quantitatifs simultanément.

1 Les auteurs reconnaissent que certaines activités développées par les migrants ne peuvent être qualifiées de transnationales que dans un sens assez large. Il s'agit, pourtant, de pratiques qui ont un rôle important dans l'entretien et l'intensification des rapports entre les migrants et leur pays d'origine, et qui s'inscrivent, donc, dans la logique du transnationalisme. 


\section{CARACTÉRISTIQUES GÉNÉRALES DE L'IMMIGRATION CAPVERDIENNE AU PORTUGAL ET DES PORTUGAIS EN SUISSE}

\section{L'immigration portugaise en Suisse}

Au cours de la vague migratoire portugaise vers les pays industrialisés de l'Europe, qui a eu lieu dans les années 1960 et au début des années 1970, la Suisse n'est apparue que très marginalement comme destination pour les émigrants portugais ${ }^{2}$. Cette situation a changé significativement à partir du milieu des années 1980, lorsque ce pays est devenu la première destination pour les émigrants portugais (Marques, 2006). Entre 1984 et 2006, le solde migratoire des Portugais qui sont entrés en territoire helvétique avec un permis de séjour annuel ou permanent a été de 118419 individus (Marques, 2008) . $^{3}$.

Suite à cette immigration, les Portugais sont devenus la troisième communauté d'étrangers résidant en territoire helvétique, après les Italiens et les Serbes ${ }^{4}$. À la fin de décembre 2006, 173477 Portugais résidaient en Suisse, dont 122935 (70,8 \%) étaient porteurs d'une autorisation d'établissement. La politique migratoire suisse traditionnellement axée sur la rotation de la main-d'œuvre n'a laissé aux émigrants portugais que l'option d'une migration saisonnière qui ne s'est transformée que lentement en une émigration permanente.

Les migrants portugais en Suisse présentent certaines caractéristiques semblables à celles des migrants portugais qui, dans les années 1960 et au début des années 1970, se sont installés dans d'autres pays industrialisés de l'Europe du Nord : à savoir un flux de main-d'œuvre composé essentiellement de jeunes travailleurs. Toutefois, contrairement au courant migratoire intra-européen précédant la crise pétrolière et économique de 1973/74, le flux migratoire vers la Suisse a toujours présenté une forte composante féminine qui est indépendante du processus du regroupement familial. L'insertion des immigrés portugais dans les secteurs d'emploi fortement dépendants de la main-d'œuvre étrangère (bâtiment, hôtellerie et restauration) et le manque conjoncturel d'emploi au Portugal confortent l'hypothèse qu'il s'agit, comme pour l'émigration des années 1960 et 1970, d'un transfert international de main-d'œuvre qui, cependant, s'étend maintenant aux deux sexes. Dans ce sens, la migration féminine devient plus visible et diversifiée dans la mesure où elle est tout autant migration de travail que mouvement lié au regroupement familial. Les femmes apparaissent comme des protagonistes actives de la migration, donnant lieu à des

2 En 1960, 373 Portugais résidaient en Suisse, en 1975, 5996.

3 Entre 1984 et 2006 sont entrés en Suisse 250649 Portugais avec un permis de séjour annuel ou permanent. Pendant la même période 132210 Portugais possédant des permis ont quitté la Suisse.

4 Les Portugais présents en Suisse représentent 11,4 \%, les Serbes 12,5\% et les Italiens 19,1\%. 
formes migratoires considérées généralement comme marginales par rapport à un modèle migratoire dominant, où la migration féminine est subordonnée à la migration masculine.

Les projets migratoires des émigrants portugais en Suisse sont, dans la perspective de ceux-ci, considérés, généralement, comme temporaires et orientés vers leur pays d'origine. C'est cette « orientation vers l'origine » qui structure les rapports sociaux et les activités économiques des émigrants, en déterminant un vécu spatialement et temporellement bipolaire. Premièrement, les migrants se trouvent pris entre deux espaces géographiques où leurs projets personnels s'organisent. Au plan économique, le rapport entre ces deux espaces (le pays de destination et le pays d'origine) se fonde sur le salaire, perçu en territoire helvétique, et sur l'épargne destinée à rendre possible la consommation dans le pays d'origine. En outre, la nature instrumentale de la migration (considérée comme une source de financement des projets individuels et familiaux de l'émigrant), réactive constamment la distinction entre le présent (le temps migratoire) et l'avenir (le temps du retour), et exerce par là même une influence décisive sur l'insertion des Portugais dans la société helvétique. D'une part, elle réduit les efforts d'intégration des émigrants : le caractère provisoire de la migration ne justifie pas, selon le point de vue rationnel de l'émigrant, un plus grand engagement avec la population locale, ni l'apprentissage de la langue et des mœurs en vigueur dans la société d'accueil. D'autre part, le raisonnement économique sous-jacent à la définition du projet migratoire comme transitoire amène l'émigrant à essayer de maximiser son gain lors de son séjour en territoire helvétique. La réalisation d'heures supplémentaires, le pluri emploi et le changement fréquent d'emploi sont des exemples de comportements adoptés par les émigrants avec l'intention de rentabiliser financièrement leur séjour en Suisse (Marques, 2006).

\section{Les migrants capverdiens au Portugal}

Les origines de l'immigration capverdienne au Portugal peuvent être trouvées dans les années 60, quand un nombre significatif de travailleurs capverdiens migrent vers celle qui était, alors, la métropole pour travailler dans le secteur minier, le bâtiment et les travaux publics. Ces Capverdiens sont embauchés au Portugal comme main-d'œuvre de substitution, pour remplacer la main-d'œuvre locale émigrée vers d'autres pays européens (ex. : la France ou l'Allemagne) ou pour compenser l'absence des soldats envoyés combattre la guerre coloniale. À leur manière, ils ont fonctionné comme des travailleurs invités et remplacé les émigrants portugais, qui sont devenus des guest-workers dans les pays de l'Europe Centrale ou du Nord. Ces immigrants originaires du Cap-Vert se sont insérés dans les secteurs de l'économie qui, à cette époque-là, étaient les plus dépourvus de main-d'œuvre, à savoir pour la plupart dans le secteur du bâtiment et travaux publics. La population capverdienne se concentre dans les districts de Lisbonne et de Setúbal, qui à eux seuls réunissent le 85-90\% environ de la population capverdienne résidant au Portugal. 
Figure 2 : Citoyens capverdiens légalement résidant au Portugal 1980-2006

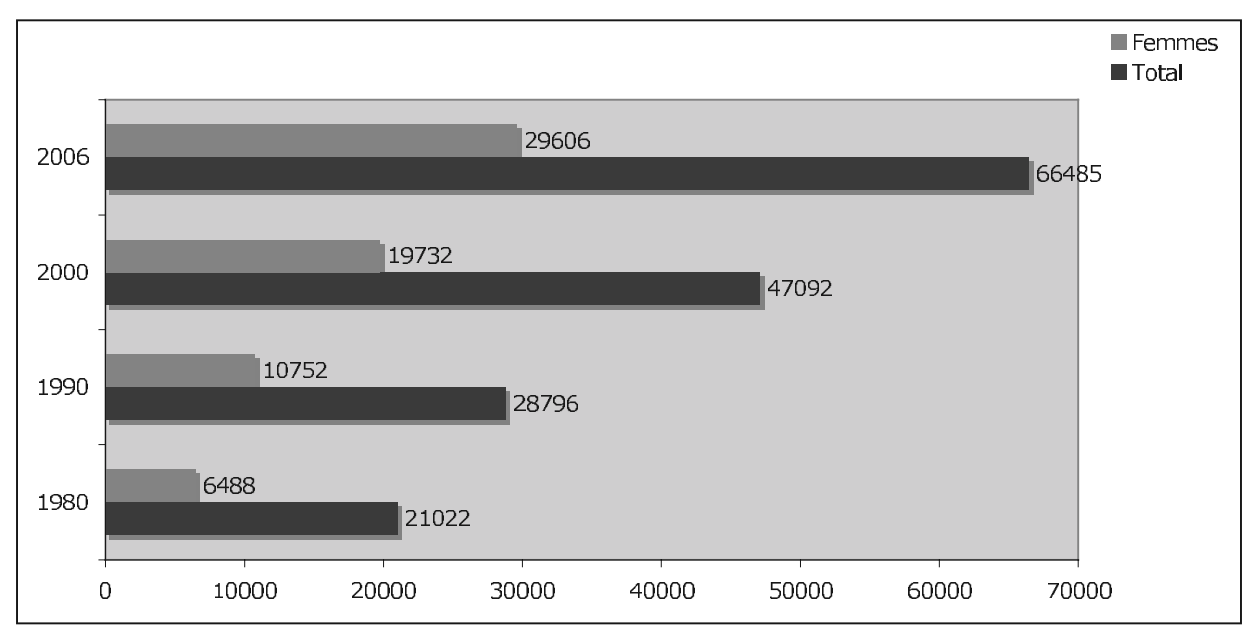

Source : Estatísticas Demográficas Sef. * 2006 nombres non définitifs. Il faut en 2006 additionner les 8574 Permis de séjour.

Les Capverdiens d'origine et leurs descendants nés au Portugal constituent un groupe hétérogène résultant d'une somme de flux successifs, ayant chacun une histoire propre d'insertion socio-économique. $\mathrm{Si}$, comme nous avons vu, le premier flux date des années 1960, c'est au cours des décennies postérieures que la communauté s'est développée. Dans les années 70, l'indépendance des colonies portugaises en Afrique produit un important mouvement de rapatriement vers la métropole, qui intéresse également les Capverdiens. Parmi eux nombreux sont ceux qui ont la nationalité portugaise $^{5}$. Ils se concentrent dans la région métropolitaine de Lisbonne et, d'une façon générale, ils s'insèrent sur le marché du travail, généralement dans des secteurs comme le bâtiment et les travaux publics, les services de nettoyage industriel et/ou domestique ou le colportage. Ce mouvement migratoire constitue la deuxième vague des migrations capverdiennes vers le Portugal. La troisième vague commence dans les années 1980 et, d'une certaine manière, elle inaugure une nouvelle phase dans l'immigration au Portugal, marquée par une forte prédominance des flux internationaux de travail sur les mouvements liés au regroupement familial. Les caractéristiques de l'insertion dans l'espace et le travail n'ont pas changé ; cette troisième vague est caractérisée par un équilibre entre les sexes dans l'immigration capverdienne, qui se maintient jusqu'à aujourd'hui.

5 Le Décret-loi $n^{\circ} 308-\mathrm{A} / 75$ du 24 juillet 1975 a retiré rétroactivement la nationalité portugaise à un grand nombre de ces migrants capverdiens, en les transformant en immigrants. Cette Loi laisse, pourtant, ouverte la possibilité de maintenir ou d'obtenir la nationalité portugaise au titre de son article 5. Postérieurement, la loi de la Nationalité (Loi n 37/81 du 3 octobre) a modifié le principe d'attribution de la nationalité du jus soli en faveur du jus sanguinis. Cependant, cette Loi n'a été abrogée qu'en 1988. 
L'immigration capverdienne vers le Portugal synthétise d'une manière particulièrement précise la complexité du processus post-colonial et la transition du Portugal à un pays aussi d'immigration. Depuis quelques décennies, cette immigration réunit des types distincts de migrants qui, dans leur totalité, ne forment pas une communauté homogène ; il s'agit plutôt d'un ensemble qui reproduit au Portugal les différentes structures de classe, de statuts, d'âge, de sexe, etc. du pays d'origine. Le nombre de migrants capverdiens (et leurs descendants) résidant au Portugal est mal connu, mais il est, évidemment, supérieur au nombre d'individus de nationalité capverdienne résidant au Portugal : on peut l'estimer entre 100000 et 200000 individus, si l'on prend en compte ceux qui sont nés au Cap-Vert, ceux qui détiennent la nationalité capverdienne et les descendants de Capverdiens. Les projets migratoires des immigrants capverdiens au Portugal sont, dans la perspective de ceux-ci, considérés, généralement, comme temporaires à long terme et orientés vers le retour à leur pays d'origine seulement après la retraite. Toutefois, en pratique, le Portugal finit par fonctionner comme un lieu d'installation définitive et même comme un lieu de retraite pour beaucoup de Capverdiens qui, lorsqu'ils quittent la vie active dans les pays européens où ils étaient immigrés, préfèrent s'installer au Portugal au lieu de rentrer au Cap-Vert de façon permanente (Moldes-Farelo, s.d.).

\section{TRANSNATIONALISME : VIEILLES OU NOUVELLES PRATIQUES ?}

Au cours des deux dernières décennies, plusieurs auteurs ont réfléchi sur le transnationalisme ${ }^{6}$ en tant que phénomène émergent ou comme un fait classique des migrations qui prend actuellement une prépondérance plus grande qu'aux époques précédentes (Itzigsohn et Saucedo, 2002). L'usage d'expressions telles que « communautés transnationales », «pratiques transnationales », "immigrants transnationaux » s'est généralisé, dans la plupart des cas, sans un support empirique suffisamment étayé. Malgré cela, l'importance que ce nouveau regard sur les migrations et les immigrants a pris graduellement ne peut être nié pour la compréhension des mouvements migratoires contemporains, basée, cette fois, sur une logique d'analyse multidirectionnelle complexe. En fait, au cours des deux dernières décennies, on a assisté à un changement dans le paradigme de l'étude des migrations, l'analyse des communautés d'immigrants et leurs descendants et la façon dont ils interagissent avec les sociétés où ils s'insèrent. Le modèle du transnationalisme oblige à la transformation d'une logique d'analyse de relations unidirectionnelles simples (origine-destination, migration de retour, (re)groupement familial, migrations temporaires ou définitives, etc.) à une autre, plus complexe, qui comprend des migrations circulaires, la réémigration, les transmigrations, les migrations transfrontalières, les communautés transnationales, les pratiques transnationales, etc.

6 Selon ce nouveau paradigme, les immigrants redéfinissent, mais n'abandonnent pas les liens qui les unissent à leur pays d'origine. Il n'existe pas un processus d'assimilation/incorporation totale dans les pays de destination, mais plutôt un partage complexe entre les deux sociétés créant ainsi une multiplicité de liens qui transcendent les frontières nationales et rendent plus complexes les relations sociales avec les sociétés d'origine et de destination. 
Si l'on ne dispose que d'exemples isolés et limités de transnationalisme, cela est dû au fait que le concept a été élaboré avant tout à l'intérieur de la discipline anthropologique ${ }^{7}$ qui privilégie les études de cas $^{8}$. Ces exemples sont déjà suffisants pour construire des typologies préliminaires et pour créer de nouvelles catégorisations, mais ils ne permettent pas d'évaluer l'étendue et l'évolution du transnationalisme en tant que fait social. D'autre part, le fait que les pratiques transnationales sont, selon la plupart des études réalisées, surtout un phénomène de la première génération des migrants et qu'elles diminuent ou disparaissent au cours des générations suivantes transforme le transnationalisme en une étape intermédiaire du processus d'assimilation. Finalement, le transnationalisme ne semble pas être un nouveau phénomène ${ }^{9}$, mais il apparaît comme différent du «vieux » transnationalisme à cause de l'influence de la mondialisation (Foner, 1997).

La réduction de la dimension relative du monde, qui résulte de la contraction des distances-temps et des distances-coûts et la facilitation des flux de communication permettent aux migrants de maintenir un contact plus fréquent et plus dense avec les régions d'origine dans une nouvelle géographie multipolaire. Dans ce nouveau mode d'organisation, un réseau de réseaux, le lieu, la région ou le pays d'origine joue un rôle de référence centrale, réelle ou imaginaire, mais la logique des relations et des processus sociaux devient plus complexe, dans la mesure où chaque lieu du réseau se rapporte soit au point d'origine, soit à d'autres lieux de destination (Malheiros, 2001). D'autre part, le potentiel de contacts, d'échanges et de multi dépendances s'intensifient et se démocratisent, une fois qu'un nombre croissant d'individus peuvent y accéder plus facilement.

Si nous nous limitons au concept de «pratiques transnationales » et à sa construction en tant qu'objet dans les sciences sociales, il semble qu'il y a encore quelques lacunes du point de vue des apports théoriques qui se traduisent par une absence relative de connaissance empirique sur les pratiques transnationales.

Les études de cas réalisées sur différents groupes migrants révèlent que souvent seul un nombre restreint de migrants s'engage dans des pratiques transnationales dans le cadre économique ou politique alors que la grande majorité ne s'y

7 Le terme transnational n'est pas récent et il est utilisé, par exemple, dans le titre d'un article classique dont l'auteur est Randolph S. Bourne, cité par Portes, publié en 1916, intitulé Transnational America. Ensuite, le concept a été utilisé de façons diverses, en se rapportant, en particulier, aux activités des entreprises globales (Portes, $2006: 208$ ).

8 Basées sur des approches ethnographiques, les études sur le transnationalisme présentent des avantages et des limites, de sorte que plusieurs auteurs insistent sur l'utilité de réaliser des études comparatives et quantitatives.

9 Il est évident que les immigrants ne sont pas les seuls acteurs transnationaux et que tous les immigrants ne sont pas transnationaux, ce qui contrarie, ainsi, une certaine notion initiale des études anthropologiques qui annonçaient la découverte «d'une nouvelle roue ». Des études ont montré que seulement une minorité des immigrants et leurs descendants se caractérise par un engagement régulier dans des activités transnationales et que l'engagement occasionnel luimême ne constitue pas une pratique universelle. 
engage qu'occasionnellement. De façon complémentaire, certaines études ont exploré le rapport entre les migrations et le développement, en définissant leur caractéristique transnationale comme un produit du capitalisme avancé ; il exerce son influence sur les pays non industrialisés ou périphériques, en les faisant dépendre des envois d'argent des émigrants eux-mêmes ; ces liens entre le pays de destination et le pays d'origine tissent de nouvelles relations de dépendance.

La discussion entre différentes activités attribuées au transnationalisme des immigrants est polarisée entre les pratiques «from below» et les pratiques «from above » (Smith et Guarnizo, 1999). Dans nos recherches, nous avons choisi une division entre les pratiques transnationales collectives et les pratiques transnationales individuelles, toutes les deux pouvant être génériquement caractérisées comme «from below ». Les acteurs transnationaux (individuels ou collectifs) développent des formes actives de participation à des processus économiques, politiques, civiques et culturels dans le lieu, la région ou le pays d'origine, ainsi que dans le lieu, la région ou le pays de destination. Nous excluons de notre analyse la dimension symbolique, celle qui unit émotionnellement les différents individus aux lieux distincts où ils ont été socialisés, souvent mentionnés comme un lien permanent. Nous tenons compte toutefois du fait que cette liaison symbolique se traduit par des consommations spécifiques : gastronomie, danse, littérature, musique, tenues et modes vestimentaires, etc., qui relient l'origine et la destination (et, parfois, d'autres noyaux migratoires) et ce sont celles-ci qui sont vraiment capables de constituer des pratiques transnationales. À travers ces consommations, il devient possible de créer des univers symboliques transnationaux, des " communautés de sentiments », des identités prospectives, un partage de goûts, de plaisirs et d'aspirations (Appadurai, 1996) qui, à leur manière, relient le lieu, la région ou le pays d'origine au lieu, région ou pays de destination. Les pratiques transnationales détectées se distribuent selon des sphères distinctes (politique, culturelle, économique, etc.) ; les frontières entre elles étant, parfois, fluides et l'attribution d'une certaine pratique à l'une ou l'autre sphère étant sujette à discussion.

\section{PRATIQUES TRANSNATIONALES CONCRÈTES DES IMMI- GRANTS : LES PORTUGAIS ET LES CAPVERDIENS}

Le transnationalisme n'est pas un phénomène récent ni pour les Capverdiens (Meintel, 2002) ni pour les Portugais. C'est un phénomène qui a des racines dans l'émigration du début du $\mathrm{XX}^{\mathrm{e}}$ siècle pour des raisons de travail des deux pays, qui a maintenu certaines de ses pratiques traditionnelles au cours du siècle et qui, d'une certaine façon, s'est renouvelé avec les outils que la mondialisation a mis à sa disposition. La plupart de ce type de pratiques est aussi observable parmi les émigrants portugais, même si elles présentent des caractéristiques et des degrés d'intensité différents selon les différents flux de l'émigration portugaise. Dans le cas des émigrants portugais résidant dans les pays européens, on peut penser que l'intensification des liens sociaux, culturels et économiques avec leur pays d'origine a entraîné, au cours du temps, le développement d'un ensemble de pratiques qui peuvent, actuellement, être classées comme «transnationales ». Il s'agit, pour l'essentiel, d'un 
ensemble d'activités basées sur l'entretien de contacts plus ou moins réguliers entre les émigrants et, surtout, leurs lieux ou régions d'origine, facilités par la communication presque instantanée que les nouvelles technologies de l'information permettent. La définition de ces pratiques comme transnationales devient particulièrement évidente si nous adoptons une définition englobante de transnationalisme, telle que celle que Grosfoguel et Cordero-Guzmán suggèrent; elle comprend soit les migrants qui entretiennent des relations multiples à travers une forte circulation physique entre le pays d'origine et le pays de destination, soit ceux qui entretiennent ces relations par d'autres moyens (l'envoi d'argent, l'envoi de biens, les contacts avec le pays d'origine, etc.) (Grosfoguel, 1998). Au fond, il s'agit d'élargir la définition de transnationalisme proposée par Vertovec :

"The actual ongoing exchanges of information, money, or resources, as well as regular travel and communication, that members of a diaspora may undertake with others in the homeland or elsewhere within the globalised ethnic community. Diasporas arise from some form of migration, but not all migration involves diasporic consciousness; all transnational communities comprise diasporas but not all diasporas develop transnationalism »(Vertovec, 2001).

\section{Pratiques transnationales dans la sphère économique}

Si l'on considère les relations des Capverdiens avec leur pays d'origine, via téléphone/téléphone portable, lettre, e-mail, envois financiers ou autres et envoi de colis, il apparaît que la grande majorité des immigrants capverdiens au Portugal est engagée dans des activités transnationales occasionnelles. Parmi celles-ci, on relève, parce que plus faciles à quantifier, les envois financiers formels destinés à l'entretien des familles restées dans l'archipel ${ }^{10}$. Cependant, un pourcentage élevé de ces envois dépend d'autres acteurs transnationaux (le «bon porteur» de lettres, le parent ou le compatriote qui transporte un colis) dans une intéressante double pratique transnationale. Ces pratiques sont duelles et complémentaires entre le formel et l'informel, les secondes pouvant être identifiées, mais non pas quantifiées. Les envois de fonds des émigrants constituent un facteur très important pour l'équilibre économique de beaucoup de familles au Cap-Vert tout en participant à la réduction du déficit de la balance des paiements courants de l'archipel. L'évolution de ces envois financiers formels peut être analysé dans le tableau suivant, en comparant différents pays. Le Portugal, au cours des dernières années, est devenu le principal pays de provenance d'envois d'argent pour le Cap-Vert.

Les virements bancaires pour l'achat de terrains ou d'habitation est la principale pratique transnationale. De façon complémentaire, les versements aux banques du pays ont encore un certain poids, mais d'autres investissements, comme la construction d'une maison ou l'investissement dans l'immobilier, le commerce ou

10 L'envoi régulier d'argent est une variable dépendante de l'existence de familiers à charge. Au fur et à mesure du regroupement familial ou de l'émigration d'autres membres du ménage, les envois tendent à diminuer en termes de périodicité et de montant. 
l'industrie ont un poids moindre. Quelques exemples d'investissement dans des affaires de portée locale (des entrepreneurs qui s'engagent dans le marché des actions, débutant du Cap-Vert, la création d'entreprises ou des joint-ventures avec des entreprises locales) apparaissent mais ils ne sont pas généralisables. Récemment, des domaines d'affaires spécifiques ont été créés (banques ou entreprises de promotion immobilière) destinées à capter l'épargne des Capverdiens à l'extérieur (fonds d'investissement dans le tourisme et l'immobilier, acquisition de logement locatif), mais les effets sont encore limités.

Comme pour les Capverdiens du Portugal, la plupart des émigrants portugais en Suisse participe à des pratiques transnationales à caractère économique, en particulier, celles qui relèvent de l'envoi d'argent au pays via leurs comptes bancaires, pour l'investissement immobilier ou pour le soutien de la famille restée au pays (particulièrement, pour le conjoint, les enfants ou les parents). Contrairement à ce qui se passe dans le cas des Capverdiens, l'argent est envoyé en général par des voies formelles (les banques et les bureaux de poste), les banques portugaises ayant développé un réseau d'agences et de représentants actifs sur le territoire helvétique et spécialisé dans l'acheminement de l'épargne des émigrants vers leurs comptes bancaires au Portugal ${ }^{11}$.

Les envois financiers destinés à l'investissement immobilier méritent une attention particulière. Ce type d'investissement a connu une croissance importante depuis la fin des années 1990, en partie à cause de l'action des agents immobiliers portugais, qui se sont déplacés en Suisse avec l'intention d'attirer les investissements des migrants dans les projets d'urbanisation en chantier. Dans un grand nombre de cas, la motivation sous-jacente à ces investissements est de nature économique, car elle vise l'utilisation de l'épargne dans des produits offrant une meilleure rémunération du capital investi que les versements bancaires traditionnels. Malgré l'importance des deux pratiques que nous venons de mentionner dans la constitution d'espaces sociaux et économiques où les migrants relient leur pays d'origine à leur pays de destination, les envois financiers dominent chez les immigrants capverdiens au Portugal ainsi que chez les Portugais en Suisse.

Les pratiques transnationales dans la sphère économique des émigrants portugais en Suisse sont encore évidentes dans un ensemble de secteurs économiques fortement dépendants de l'importation de différents types de biens portugais (les biens alimentaires, les livres, les CD/DVD, les meubles, les produits de décoration, les agences de voyage, etc.). La nature de ces activités, et le besoin d'exercer un contrôle effectif sur les produits à importer, impliquent des voyages fréquents entre la Suisse et le Portugal et l'entretien d'une relation de proximité avec les producteurs et les fournisseurs des produits.

11 La nature formelle des envois d'argent permet de savoir que les montants envoyés par les émigrants portugais en Suisse ont dépassé les $576381000 €$, en 1996, pour atteindre les $721211000 €$, en 2001, et les $530720000 €$, en 2006 (source : Banco de Portugal, plusieurs années). 
Les pratiques que nous venons de mentionner montrent comment les migrants contribuent à la constitution d'espaces sociaux et économiques reliant leur pays/région d'origine au pays d'accueil. Il s'agit d'activités qui, en général, sont assez régulières. Elles sont individuelles et familiales essentiellement et ne vont pas au-delà des membres du réseau familial du migrant. Des activités qui impliquent un réseau plus étendu d'acteurs et qui cherchent, à travers une action collective, à contribuer au développement du pays/région d'origine ou à soulager les effets des désastres naturels (comme les incendies ou les inondations) sont moins fréquentes et sont peu structurées.

\section{Pratiques transnationales dans la sphère socioculturelle}

Alors que la culture n'a pas été considérée comme une variable explicative dans la sociologie des migrations, c'est toutefois dans la sphère culturelle que les pratiques transnationales des migrants portugais ou capverdiens sont visibles. L'entretien des liens avec le pays d'origine se fait surtout à travers l'utilisation de la langue d'origine, la gastronomie, la consommation ou la musique, les médias ou la littérature du pays d'origine qui sont valorisés autant si non plus que ceux du pays d'accueil.

En ce qui concerne les pratiques transnationales des Capverdiens au Portugal, la dynamique des productions et/ou des consommations culturelles est peut-être, l'aspect le plus saillant du transnationalisme capverdien. Malgré sa population réduite, le Cap-Vert est un pays qui a une visibilité musicale élevée. Des artistes capverdiens sont connus dans le cadre de la «world music ». Ils circulent entre les pays ou les villes qui accueillent des immigrants capverdiens (Esteves et Caldeira, 2001). Les producteurs et les consommateurs peuvent se trouver, de manière indifférenciée, au Portugal, au Cap-Vert ou dans d'autres pays ${ }^{12}$. Une grande partie de la production musicale d'origine capverdienne est réalisée par des personnes d'origine capverdienne qui vivent en dehors du Cap-Vert (Cidra, 2005) ; la production et la consommation de musique capverdienne relèvent de pratiques transnationales de haute intensité (Góis, 2006). Lisbonne est une scène importante de pratiques culturelles qui se prolongent dans d'autres domaines, comme celui de la langue. En rendant possible une interaction entre des cultures locales d'où viennent les immigrants et les cultures des lieux d'installation, la musique, mais aussi la langue dans une moindre mesure, permet de comprendre la logique du transnationalisme comme un territoire unique d'action sociale où les immigrants se meuvent dans différentes cultures et systèmes sociaux (Brettell et Hollifield, 2000).

Les Portugais en Suisse ont plutôt tendance à consommer des produits médiatiques portugais : la télévision, mais aussi une certaine presse et des journaux sportifs. L'existence d'une chaîne publique portugaise, dont les émissions sont destinées aux communautés portugaises dans le monde, ainsi que l'abonnement à un service de télévision émis par satellite, ont élargi les opportunités des Portugais à

12 Le marché musical portugais est, immédiatement après le Cap-Vert, celui qui consomme le plus de musique capverdienne. 
l'extérieur de maintenir et d'intensifier, bien qu'en qualité de récepteurs, les liens avec leurs pays d'origine. Les trois moyens de communication, télévision, radio et journaux, semblent, à des degrés divers, susciter l'intérêt de la première génération d'émigrants portugais, tandis que les choix médiatiques des jeunes issus de la migration s'orientent plutôt vers des produits médiatiques plus attractifs pour leur groupe d'âge, indépendamment de l'origine nationale et de la langue dans laquelle ils sont transmis. Comme plusieurs interviewés l'ont dit, l'ensemble des activités développées par les associations d'émigrants ayant pour objectif de maintenir les liens avec le Portugal ${ }^{13}$ continuent à avoir une certaine importance pour la première génération d'émigrants, alors qu'elles n'attirent plus la deuxième génération ${ }^{14}$. La multiplication des lieux qui offrent des activités similaires à celles qui n'étaient offertes auparavant que par les associations ont contribué à la diminution de leur rôle dans le maintien des liens avec le pays et réduit la participation des Portugais à la vie associative.

\section{Pratiques transnationales dans la sphère politique}

Pouvoir élire et être élu, participer politiquement dans le pays d'origine et dans le pays de destination, influencer des décisions dans deux États est un défi qui peut se concrétiser dans différents types de pratiques transnationales. Les Capverdiens résidents au Portugal illustrent le déficit de participation, et l'on notera qu'aux récentes élections présidentielles et législatives au Cap-Vert, celle-ci a été faible, d'une part, parce qu'il y a peu d'inscrits et d'autre part parce que le niveau d'abstention a été élevé.

La participation politique au Portugal est de deux ordres : soit les Capverdiens ont la nationalité portugaise et ils jouissent d'une pleine participation électorale, soit ils ne l'ont pas, et à condition qu'ils aient un permis de séjour, ils peuvent participer aux élections locales. Le faible niveau de participation aux dernières élections a été notable. L'analyse des entretiens réalisés confirme la faible mobilisation des Capverdiens qui est en porte-à-faux par rapport à la valeur symbolique et aux potentialités de ce type de pratiques transnationales ${ }^{15}$.

Comme pour les Capverdiens, les signes de l'existence des pratiques transnationales dans la sphère politique sont très limités chez les émigrants portugais en Suisse et restent circonscrits le plus souvent à un ensemble réduit d'émigrants politiquement actifs. L'intérêt des émigrants pour les questions politiques nationales et/ou locales est peu important. La participation électorale aux élections portugaises

13 Comme l'entretien des groupes de folklore, la promotion de différents types de festivals etc.

14 Malgré ce déclin généralisé, il faut noter que la participation de la deuxième génération aux activités associatives présente une forte variation selon la région linguistique suisse et le type d'activités développées par l'association (les activités sportives et, en particulier, la pratique du football présentent des taux de fréquentation plus élevés que les autres activités culturelles).

15 À titre d'exemple, mentionnons que le nombre de votants aux élections législatives ou aux élections présidentielles de 2006 n'a pas dépassé les 5000 dans l'Europe entière, par rapport aux 32000 électeurs inscrits environ et plus de 100000 d'électeurs potentiels. 
(parlement national et élections présidentielles) ${ }^{16}$ est faible. Malgré ce manque d'intérêt, il est possible de constater une augmentation relative de l'engagement dans les affaires de la politique nationale lorsque les thèmes en discussion sont directement liés aux communautés émigrantes ${ }^{17}$.

Le faible engagement des Portugais dans des activités politiques dirigées vers leur pays d'origine ne peut être expliqué uniquement par leur manque d'intérêt. L'insuffisance et même l'absence de structures permettant la participation politique des émigrants à l'étranger sont à souligner. En effet, bien que les partis politiques portugais aient leurs représentants en Suisse, leur action se trouve limitée par l'insuffisance de moyens financiers et humains.

\section{L'utilisation des technologies de l'information et de la communication}

L'importance du développement des nouvelles technologies de l'information et de la communication (Internet, radios en ligne, télévisions, téléphones portables) dans leurs applications les plus modernes (SMS, blogs, web pages, my space, you tube, etc.), sont utilisées par des Capverdiens émigrés, qui les utilisent pour entretenir des liens entre nations.

Les nouvelles technologies jouent un rôle dans la manière dont les pratiques transnationales se structurent: dans le domaine politique avec l'envoi de SMS aux électeurs potentiels, dans le domaine culturel avec la création de blogs consacrés à la musique de l'archipel, dans le domaine économique avec la réalisation de contacts commerciaux, dans le domaine social avec l'utilisation de l'Internet. On a constaté que les nouvelles technologies restauraient et, en même temps, élargissaient les réseaux de contacts. C'est par cette voie que, par exemple, les communautés les plus anciennes, telles que celles des Capverdiens aux États-Unis, en Argentine ou au Sénégal, où certains des premiers émigrants avaient déjà perdu les contacts avec leur origine, peuvent renouer des contacts avec le Cap-Vert ou avec des Capverdiens émigrés dans d'autres pays.

Ces technologies de l'information et de la communication contribuent à une activation des réseaux sociaux solidaires à longue distance, l'une des caractéristiques du transnationalisme contemporain, capables de mobiliser les immigrants et de les transformer en des médiateurs entre les sociétés d'origine et de destination. Si l'utilisation des applications modernes des technologies de l'information reste peu accessible aux émigrants de la première génération par contre les luso-descendants et une partie des Portugais arrivés plus récemment en Suisse, utilisent ces technologies

161750 des 4408 émigrants inscrits comme votants aux consulats ont voté aux élections législatives de 2005 ; sur 5730 inscrits, 871 ont voté aux élections présidentielles, (Source : STAPE, disponible en ligne sur : http://www.stape.pt/eleiref/index_eleiref_nt.htm).

17 En 2003/2004, dans la discussion autour du projet du Gouvernement portugais de supprimer les émissions internationales de la chaîne de télévision publique, et en 2005/2006, dans le débat autour de la restructuration du réseau consulaire portugais. 
pour maintenir des liens avec leur pays d'origine ou leurs parents/amis émigrés dans d'autres pays ${ }^{18}$. Selon nos interlocuteurs, cette utilisation est plus fréquente après une période de vacances au Portugal ou lors d'évènements festifs (les anniversaires, Noël, etc.).

\section{DISCUSSION : TRANSNATIONALISME INDIVIDUEL/FAMILIAL PLUTÔT QUE COMMUNAUTAIRE}

Le transnationalisme capverdien se fonde sur une base familiale alors que la famille est une valeur centrale [la famille est «étendue » et «(re)construite » 19 , basée non seulement sur l'ascendance commune, mais aussi sur le vécu en commun $(\text { Malheiros, 2001 })^{20}$ ]. En cela il rejoint ce qui a déjà été identifié dans d'autres groupes migrants (Bryceson et Vuorela, 2002). Dans un classique des études sur le transnationalisme, Bash et ses collaborateurs affirment :

"The family is the matrix from which a complexly layered transnational social life is constructed and elaborated. [It] facilitates the survival of its members, their class formation and mobility; and as the repository of cultural practices and ideology shaped in the home society, it mediates identity formation in the new setting as it socializes its members into a transnational way of life » (Basch et al., 1994: 79).

Dans le cas des migrations capverdiennes, les réseaux sociaux organisés autour de la famille, du lieu d'origine, de l'île d'origine, du pays d'origine, ou de la « nation ethnique » imaginée, structurent et conditionnent, à des niveaux différents, le processus migratoire et les pratiques transnationales. Un bon exemple peut être trouvé dans ce que Jorge Malheiros (2001) a caractérisé comme une culture migratoire active consolidée dans un «savoir circulaire » entre les différents noyaux migratoires en Europe. Dans un travail sur les communautés capverdiennes à Lisbonne et à Rotterdam, il montre que les initiatives transnationales les plus courantes se situent sur le plan individuel et familial (Malheiros, 2001). Ces initiatives ont lieu, le plus souvent, du lieu de destination vers le lieu d'origine, raison pour laquelle nous devrions, peut-être, utiliser le terme « translocalités », proposé par Michael Smith et Luís Guarnizo (1999), plutôt que pratiques transnationales.

Les activités transnationales des Portugais en Suisse deviennent, également, plus visibles dans le cadre individuel et familial. La famille a un rôle central dans le

18 La communauté portugaise en Suisse ne présente pas des patrons d'activité homogènes, des différences d'utilisation selon l'instruction, la région linguistique et l'âge pouvent être trouvées.

19 Familles élargies, avec enfants et autres parents consanguins ou par alliance vivant en proximité étroite ; familles recomposées, avec enfants issus de mariages précédents.

20 Ce concept de famille se fonde sur les divers types de liens sociaux émergeant de différentes formes d'unions conjugales, mettant en œuvre les pratiques éducatives concernant les enfants et le maintien de liens consanguins intra et intergénérationnels étroits. La forte inclusivité de cette notion de famille confère à ses frontières un caractère extrêmement flexible. 
Tableau 1 : Pratiques transnationales des émigrants portugais et capverdiens

\begin{tabular}{|c|c|c|c|c|}
\hline & $\begin{array}{c}\text { Capverdiens } \\
\text { Individuelles/ } \\
\text { Familiales }\end{array}$ & Collectives & $\begin{array}{c}\text { Portugais } \\
\text { Individuelles/ } \\
\text { Familiales }\end{array}$ & Collectives \\
\hline $\begin{array}{l}\text { Sphère } \\
\text { économique }\end{array}$ & $\begin{array}{l}\text { - Envoi d'argent et inves- } \\
\text { tissement (habitation, } \\
\text { affaires et terrains) } \\
\text { - Envoi de biens divers } \\
\text { - Emprunts dans le cadre } \\
\text { familial } \\
\text { - Versements bancaires } \\
\text { dans le pays d'origine } \\
\text { - Envoi de billets d'avion } \\
\text { ou aide à l'achat }\end{array}$ & $\begin{array}{l}\text { - Organisation } \\
\text { de fêtes } \\
\text { religieuses, } \\
\text { de festivals } \\
\text { musicaux }\end{array}$ & $\begin{array}{l}\text { - Envois d'argent, } \\
\text { - Investissements } \\
\text { immobiliers, } \\
\text { activités d'entreprise }\end{array}$ & $\begin{array}{l}\text { - Initiatives } \\
\text { sporadiques de } \\
\text { collecte de } \\
\text { fonds pour des } \\
\text { projets locaux } \\
\text { dans le pays } \\
\text { d'origine (des } \\
\text { fêtes, la } \\
\text { rénovation de } \\
\text { l'église, etc.) } \\
\end{array}$ \\
\hline $\begin{array}{l}\text { Sphère } \\
\text { socioculturelle }\end{array}$ & $\begin{array}{l}\text { - Pratique de la langue } \\
\text { maternelle (créole cap- } \\
\text { verdien) ; Bilinguisme } \\
\text { - Consommation de } \\
\text { musique capverdienne ; } \\
\text { - Émigration et retour } \\
\text { - Visites régulières } \\
\text { - Associationnisme } \\
\text { (coopération et échange) } \\
\text { - Visites aux familiers [et } \\
\text { aux compatriotes] dans } \\
\text { des pays tiers }\end{array}$ & $\begin{array}{l}\text { - Circulation de } \\
\text { musiciens et de } \\
\text { musique } \\
\text { - Circulation } \\
\text { d'écrivains et } \\
\text { de littérature } \\
\text { - Création de } \\
\text { maisons d'édi- } \\
\text { tion musicale } \\
\text { - Organisation et } \\
\text { participation à } \\
\text { des tournois } \\
\text { sportifs de base } \\
\text { - Mariages mixtes }\end{array}$ & $\begin{array}{l}\text { - Pratique de la langue du } \\
\text { pays d'origine, fréquen- } \\
\text { tation des cours de } \\
\text { langue et culture } \\
\text { portugaise par les enfants } \\
\text { des émigrants } \\
\\
\end{array}$ & $\begin{array}{l}\text { - Associations } \\
\text { d'émigrants } \\
\text { - Consommation } \\
\text { de produits } \\
\text { médiatiques } \\
\text { portugais }\end{array}$ \\
\hline Sphère politique & $\begin{array}{l}\text { - Participation électorale } \\
\text { réduite, participation } \\
\text { sporadique à des } \\
\text { activités politiques }\end{array}$ & \begin{tabular}{|l|} 
- Circulation de \\
candidats aux \\
élections du \\
Cap-Vert \\
(cercles \\
d'émigration) : \\
- Organisation \\
extérieure des \\
partis du Cap- \\
Vert ; \\
- Organisation \\
des congrès \\
dans le cadre de \\
la diaspora \\
\end{tabular} & $\begin{array}{l}\text { - Participation électorale } \\
\text { réduite, participation } \\
\text { sporadique à des } \\
\text { activités politiques } \\
\\
\end{array}$ & $\begin{array}{l}\text { - Partis poli- } \\
\text { tiques portugais } \\
\text { avec des repré- } \\
\text { sentants en } \\
\text { Suisse } \\
\text { - Structures } \\
\text { d'État } \\
\text { (Consulats, } \\
\text { Ambassades) }\end{array}$ \\
\hline $\begin{array}{l}\text { Sphère de } \\
\text { l'Information/ } \\
\text { Communication }\end{array}$ & $\begin{array}{l}\text { - Contacts personnels sur l'In- } \\
\text { ternet (e-mail, programmes } \\
\text { du type messenger) } \\
\text { - Création de sites, blogs, etc. } \\
\text { - Utilisation d'espaces d'échange } \\
\text { (youtube, my space, etc.) } \\
\text { - Correspondance, appels } \\
\text { téléphoniques et envois } \\
\text { de SMS } \\
\text { - Recherche de liaisons } \\
\text { ancestrales }\end{array}$ & & $\begin{array}{l}\text { - Peu de sites portugais en } \\
\text { Suisse (quelques sites } \\
\text { personnels ou des } \\
\text { Journaux dirigés aux } \\
\text { émigrants portugais) } \\
\text { - Nombre limité de blogs } \\
\text { - Appels téléphoniques }\end{array}$ & \\
\hline
\end{tabular}


processus migratoire lui-même, à travers la consolidation des réseaux migratoires qui favorisent ce processus, ainsi que dans plusieurs aspects de la vie quotidienne du migrant. À la différence de ce qui se passe dans le cas des immigrants capverdiens, les rapports familiaux des Portugais en Suisse sont organisés autour de la famille nucléaire, la famille étendue occupant une place secondaire dans la relation du migrant à la société d'accueil et à la société d'origine.

L'importance des rapports familiaux et individuels dans l'exercice des activités transnationales par les deux groupes de migrants analysés dans cet article est schématisée dans le tableau ci-dessous. Il illustre l'hétérogénéité de l'ensemble des pratiques transnationales des migrants capverdiens et portugais.

\section{CONCLUSION}

L'entretien des liens avec leur pays d'origine constitue une caractéristique commune aux migrants. Actuellement, une partie de ces relations est interprétée par le recours à de nouvelles notions qui cherchent à refléter l'intensité, l'immédiateté et la simultanéité des relations qui se développent entre les migrants et les différentes sphères de la société d'origine.

Comme nous l'avons décrit tout au long de cet article, il y a plusieurs façons, pour les migrants, de se mettre en rapport avec leur pays d'origine (soit en termes factuels, soit en termes émotionnels ou symboliques), de la même manière qu'il y a des degrés d'engagement variables selon les différents groupes. L'analyse de leurs activités transnationales montre clairement qu'elles sont restreintes, à l'exception des pratiques spécifiques (par exemple, l'envoi d'argent), et, souvent, sporadiques. Au caractère limité des pratiques transnationales, nous devons ajouter la variabilité de ces mêmes pratiques, qui devient évidente à travers la comparaison entre les immigrants capverdiens au Portugal et les immigrants portugais en Suisse. Elle reflète, surtout, le moment et le contexte de la migration ainsi que les formes de participation des migrants à la société d'accueil et aux différentes sphères qui la constituent.

Par rapport au premier ensemble de variables (le moment et le contexte de la migration), il est à souligner que les immigrants capverdiens au Portugal et les différentes générations de leurs descendants sont le résultat de phases migratoires et de vagues migratoires distinctes. En conséquence, ils présentent une grande hétérogénéité de statuts sociaux et des modes distincts d'insertion dans la société portugaise et, par conséquent, des pratiques transnationales distinctes. Les immigrants portugais en Suisse sont, par contre, un groupe beaucoup plus homogène qui se caractérise par une migration de travail pour la première génération et par des descendants encore majoritairement en âge scolaire. Les Capverdiens au Portugal sont le premier groupe d'origine étrangère au Portugal et les Portugais en Suisse sont la troisième communauté dans le pays. D'une manière générale, les deux groupes d'immigrants présentent des projets migratoires distincts. Les Capverdiens parient sur un projet migratoire permanent au Portugal (ou, au plus, sur une réémigration à partir du Portugal) ; les 
Portugais en Suisse s'aventurent dans un projet migratoire de moyenne ou longue durée, avec un retour projeté au Portugal. Selon les projets migratoires, les pratiques transnationales adoptées sont différentes.

La participation des migrants à la société d'accueil dans la sphère économique est une autre caractéristique qui influence le type et le mode de pratiques transnationales. Les rémunérations perçues en Suisse et au Portugal pour les mêmes professions (aide-maçon, maçon, femme de ménage, etc.) montrent que la capacité d'épargne ou d'investissement des immigrants en Suisse est supérieure à celles des immigrants au Portugal

En dépit des différences entre Capverdiens et Portugais on voit se dessiner un transnationalisme de basse intensité. S'il est certain que, occasionnellement, tous les migrants (ou presque) s'engagent dans des pratiques transnationales, peu d'entre eux partagent leur vie entre deux sociétés. La principale raison pour laquelle ils ne le font pas c'est qu'ils ne le peuvent tout simplement pas. Sur le plan symbolique par contre, une grande majorité d'immigrants mène des vies partagées entre lieu d'origine et lieu de destination. Cette dimension symbolique se concrétise dans des productions et des consommations culturelles très importantes pour les pays d'origine et de destination (la gastronomie, la musique, la langue, la littérature et la danse), qui rendent les sociétés d'accueil plus diverses et permet aux sociétés d'origine de maintenir vivantes des pratiques culturelles qui seraient condamnées à la disparition.

Une dernière remarque : l'étude du transnationalisme à partir de l'analyse des pratiques caractérisées comme transnationales rend évidente la difficulté de généraliser à tous les émigrants. Comme d'autres auteurs l'ont déjà reconnu (Portes, 2004), une fraction seulement de migrants s'engage dans des pratiques qui peuvent être considérées comme transnationales dans un sens, parfois, assez large et controversé.

Pour conclure nous pensons que nos recherches confirment que le transnationalisme et ses pratiques ne mettent pas en cause les mécanismes et les parcours d'assimilation sociale que les immigrants suivent généralement, de façon lente, mais continue. Dans différents systèmes fonctionnels (politique, économique, culturel), cette assimilation sociale est fonction du traitement différencié dont les divers groupes font l'objet. Mais cette assimilation sociale ne se produit pas sans des transformations dans la société d'origine, ainsi que dans les sociétés d'immigration, qui font que les pratiques transnationales peuvent être vues comme des maillons mettant en liens des temps et des espaces différents. 


\section{Références bibliographiques}

APPADURAI Arjun (1996) Modernity at Large: Cultural Dimensions of Globalization, Minneapolis, University of Minnesota Press.

BASCH Linda G., SCHILLER Nina Glick and BLANC Cristina Szanton (1994) Nations Unbound: Transnational Projects, Postcolonial Predicaments, and Deterritorialized NationStates, Luxembourg, Gordon and Breach.

BRYCESON Deborah Fahy and VUORELA Ulla (2002) The Transnational Family: New European Frontiers and Global Networks, Oxford / New York, Berg.

BOURDIEU Pierre (1991) Language and symbolic power, Cambridge, Mass., Harvard University Press

BRETTELL Caroline et HOLLIFIELD James Frank (2000) Migration theory: talking across disciplines, New York, Routledge.

CASTELLS Manuel et al. (2007) Mobile Communication and Society, Boston, Mass., MIT Press.

CIDRA Rui (2005) "Migração, performance e produção de fonogramas: músicos em viagem entre Cabo Verde e Portugal", Conference on Cape Verdean Migration and Diaspora, Lisboa, Centro de Estudos de Antropologia Social, 6-8 Abril 2005.

ESTEVES Alina et CALDEIRA Maria J. (2001) "Reinventing cultures: the contribution of the Cape Verdean community to the cultural dynamics of Lisbon", in Russel King (Éd.), The Mediterranean Passage, Liverpool, Liverpool University Press, 95-118.

FONER Nancy (1997) "What's new about transnationalism? New York immigrants today and at the turn of the century", Diaspora, 6 (3), 355-375.

GÓIS Pedro (2006) Emigração Cabo-Verdiana para (e na) Europa e a sua Inserção em Mercados de Trabalho Locais: Lisboa, Milão e Roterdão, Lisboa, ACIME.

GÓIS Pedro et MARQUES José Carlos (2006) "Portugal as a semiperipheral country in the global migration system", Conference on Lusophone Migration, Georgetown University, Washington DC, November 17-19, 2006.

GROSFOGUEL Ramón et CORDERO-GUZMAN Hector (1998) "International Migration in a Global Context: Recent Approaches to Migration Theory", Diaspora, 7(3), 351-368.

ITZIGSOHN José et al. (1999) "Mapping Dominican transnationalism: narrow and broad transnational practices", Ethnic \& Racial Studies, 22 (2), 316-339.

ITZIGSOHN Jose et SAUCEDO Silvia Giorguli (2002) "Immigrant incorporation and sociocultural transnationalism", International Migration Review, 36 (3), 766-798.

MALHEIROS Jorge (2001) Arquipélagos migratórios: transnacionalismo e inovação, Faculdade de Letras da Universidade de Lisboa, Dissertation de Doctorat.

MARQUES José Carlos (1997) A emigração portuguesa para a Suíça, Faculdade de Economia da Universidade de Coimbra, Dissertation de Maitrîse.

MARQUES José Carlos (2006) Os Novos Movimentos Migratórios Portugueses - O Caso da Emigração Portuguesa para a Suíça, Coimbra, Faculdade de Economia, Unversidade de Coimbra, Dissertation de Doctorat.

MARQUES José Carlos (2008) Os Portugueses na Suíça. Migrantes Europeus, Lisboa, Instituto de Ciências Sociais.

MEINTEL Deirdre (2002) "Cape Verdean Transnationalism, Old and New”, Anthropologica, XLIV, 25-42.

MOLDES-FARELO Rócio (s.d.) Inmigrantes Jubilados: La invención del ocio y la fantasía del retorno.

PORTES Alejandro (1997) "Immigration theory for a new century: some problems and opportunities", International Migration Review, 31 (4), 799-825. 
PORTES Alejandro (2004) "O estudo do transnacionalismo imigrnate", Revista Crítica de Ciências Sociais, 69, 73-93.

PORTES Alejandro (2006) "Os debates e o significado do transnacionalismo migrante", in Alejandro Portes (Éd.), Estudos Sobre as Migrações Contemporâneas. Transnacionalismo, Empreendorismo e a Segunda Geração, Lisboa, Fim de Século, 201-244.

SMITH Michael et GUARNIZO Luis (1999) Transnationalism from Below, New Brunswick and London, Transaction Publishers.

VERTOVEC Steven (2001) "Religion and diaspora", ESRC Transnational Communities Research Programme, Working Papers 01-01, Oxford. 


\section{Mondlalisations et migrations internationales}

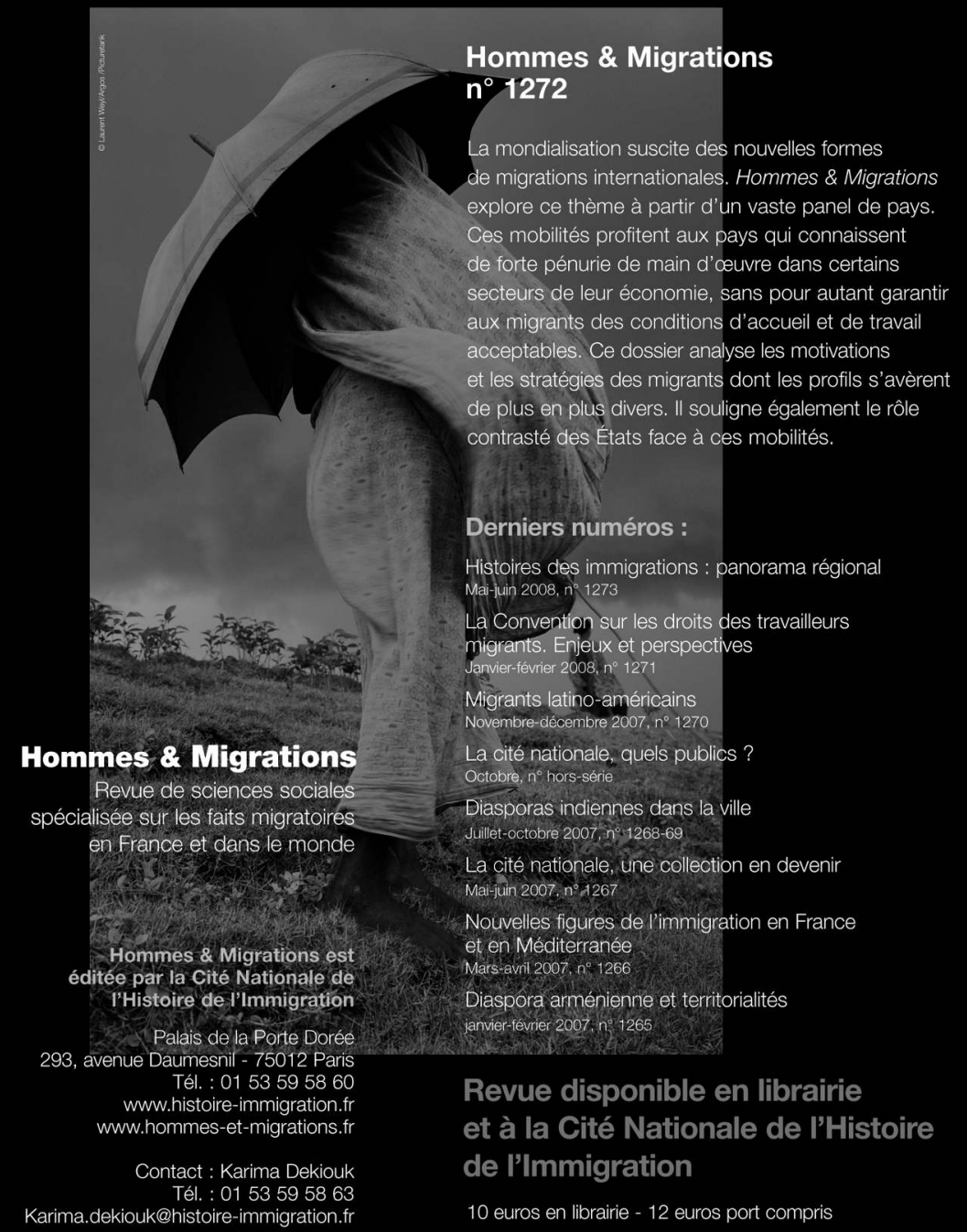

